\title{
Prognostic Value of Chemotherapy-Induced Amenorrhea in Breast Cancer: a Meta-Analysis
}

\author{
Quan-Bin Zha ${ }^{1 \&}$, Jin-Hai Tang ${ }^{2 \&}$, Xiu-Juan $\mathrm{Li}^{2}$, Lei Xia ${ }^{3}$, Zhe Zhang ${ }^{3}$, Zhao-Jun \\ $\operatorname{Ren}^{3 *}, \mathrm{Xin}-\mathrm{Yu} \mathrm{Xu}^{3 *}$
}

\begin{abstract}
Background: There is still a great deal of controversy with regard to the prognostic role of chemotherapyinduced amenorrhea (CIA) in breast cancer patients. To confirm whether CIA can serve as a useful factor in predicting clinical effects of systemic adjuvant chemotherapy, we performed this meta-analysis. Materials and Methods: Relevant studies were identified using PubMed, and Embase databases. Eligible study results were pooled and summary hazard ratios (HRs) with corresponding confidence intervals (CIs) were calculated. Subgroup analyses and an assessment of publication bias were also conducted. Results: A total of 8,333 patients from 11 published studies were identified through searching the databases. The pooled HRs for disease-free survival (DFS) suggested that CIA was associated with a significant reduction in the risk of recurrence, especially in patients with hormone receptor-positive lesions (overall $\mathrm{HR}=0.65,95 \% \mathrm{CI} 0.53-0.80, \mathrm{I}^{2}=41.3 \%$ ). When the five studies reporting the HR for overall survival $(O S)$ were pooled $(n=4193)$, a favorable trend was found $(H R=0.69$, 95\% CI 0.52-0.91, $\mathrm{I}^{2}=\mathbf{5 1 . 6 \%}$ ). No publication bias was observed in this study. Conclusions: This meta-analysis suggests that CIA predicts a better outcome in premenopausal hormone receptor-positive breast cancer patients.
\end{abstract}

Keywords: Chemotherapy - induced amenorrhea - breast cancer - meta-analysis

Asian Pac J Cancer Prev, 16 (14), 5939-5944

\section{Introduction}

Breast cancer is the most frequently diagnosed cancers and the leading causes of cancer death in women , and approximately one-third of breast cancer cases are expected to occur in women $<50$ years of age (Siegel et al., 2015). Chemotherapy has been the mainstay of adjuvant therapy for premenopausal women with node-positive breast cancer, and its effect was much higher in younger women than in older women (Yazilitas et al., 2015). The benefit of adjuvant chemotherapy has been supposed that at least part of it is derived from chemotherapyinduced ovarian ablation. Although chemotherapy and ovarian function suppression independently improve the outcome of breast cancer (EBCTCG., 2005), there is controversy about the Prognostic role of chemotherapyinduced amenorrhea (CIA) in breast cancer. Recently, a comprehensive review published in 2006 discussed the incidence of amenorrhea with various variables, its impact on survival, and dilemmas in interpreting conflicting data on CIA (Walshe et al., 2006). CIA causes a variety of climacteric problems, including hot flush, genitourinary dysfunctions, and psychological distress (Leining et al., 2006). So, a comprehensive understanding of the impact of CIA in premenopausal women is critical. Due to many discrepancies across studies including the definition of
CIA and premenopausal status, chemotherapy regimens and the characteristic of patients, we should draw a conclusion with caution. Concerning 3 articles of high quality updated in database (Jung et al., 2010; Swain et al., 2010; Park H et al., 2012), we undertook a meta-analysis to update the results and to evaluate the prognostic role of CIA in premenopausal patients with breast cancer.

\section{Materials and Methods}

\section{Literature search and selection}

We conducted a systematic literature search of PubMed and EMBASE through January 2014 by using the following key words: breast cancer, breast neoplasm, chemotherapy, drug therapy, prognostic, prognosis, and amenorrhea. References from identified studies were also reviewed. The search was limited to English language. The eligible studies should meet the following criteria: $i$ ) only original papers evaluating the association between chemotherapy-induced amenorrhea and disease free survival (DFS) and/or overall survival (OS) were selected. ii) hazard ratio (HR) for OS or DFS according to CIA either had to be reported or could be calculated from the paper. iii) for publications reported in several, only the most recent one was considered. Abstracts were excluded because of insufficient data. 


\section{Data extraction}

The following data from eligible publications was extracted respectively by two reviewers (Zha QB and Ren ZJ) with a standardized data collection form: first author's last name, year of publication, country of origin, sample size, study design, disease stage, chemotherapy regimen, median follow-up, median age, definition of CIA, main end point, adjustments for covariates, the hazard ratios (HRs) and corresponding 95\% confidence intervals (CIs). If HRs and 95\%CIs were not provided directly, estimated value was obtained indirectly by using the methods described by Tierney et al (2007). Disagreements were resolved by discussion.

\section{Statistical analysis}

The primary outcome of our meta-analysis was disease-free survival (DFS), because the sample size for OS was relatively small. When univariate and multivariate analyses of DFS and/or OS were both available, the latter was selected to be combined. The subgroup analyses were performed according to definition of CIA, hormone receptor status, premenopausal status, median follow-up, region, tamoxifen use or not, chemotherapy regimen and study design. Heterogeneity between trials was evaluated by chi-square $\left(\mathrm{x}^{2}\right)$ test and I-squared $\left(\mathrm{I}^{2}\right)$ statistic (Higgins JP et al., 2003). Statistical heterogeneity was considered significant if $p$ value less than 0.10 for the $\mathrm{x}^{2}$ test or $\mathrm{I}^{2} 50 \%$.We used a random-effects model to pool HRs. Publication bias was assessed by Begg's funnel plots and Egger's regression test (Begg et al., 1994). Additionally, We also conducted a sensitivity analysis to investigate the influence of a single trial on the overall risk (Tobias, 1999). All reported $P$ values were two-sided and $P$ values less than 0.05 were regarded as statistically significant. Statistical analyses were carried out using STATA 12.0 (Stata Corporation, USA).

\section{Results}

\section{Literature search}

We initially reviewed 355 relevant citations using search strategies as described previously. Of these, the majority were excluded after the first screening based on abstracts, because they were not relevant to our analysis, or the primary outcome was not DFS or OS. After fulltext review of 23 papers, 2 studies (Howell et al., 1984; Padmanabhan et al., 1986) were excluded because the definition of CIA was not specified. Another 6 studies (Fisher, 1979; Brincker et al., 1987; Beex et al., 1988; Richards et al., 1990; Campora et al., 1992; Borde et al., 2003) were excluded because of poor data for metaanalysis. We further excluded 2 studies (Swain et al., 2009; Ganz et al., 2011) for overlapping publication. Meanwhile, 2 studies were excluded because they were reviews (Mastro et al., 2003; Walshe et al., 2006). Finally, 11 studies (Ludwig Breast Cancer Study Group., 1985; Goldhirsch et al., 1990; Bianco et al., 1991; Budman et al., 1998; Pagani et al., 1998; Parulekar et al., 2005; Colleoni et al., 2006; Jung et al., 2010; Swain et al., 2010; Ganz et al., 2011; Park et al., 2012) were included in our meta-analysis. Flow diagram of selecting eligible trials was shown in figure $\mathrm{S} 1$.

\section{Study characteristics}

The characteristics of these 11 studies are listed in Table S1. Of these, 4 studies were conducted in North America, 4 in Europe, 2 in Korea, and 1 was a multination study. The sample size of the included studies ranged from 221 to 2341 patients. The percentage of patients becoming amenorrheic ranged from $51 \%$ (Budman et al., 1998) to $86 \%$ (Colleoni M et al., 2006). According to the definition of CIA, 5 of the 11 eligible studies defined it as cessation of menses for three months, 4 defined it as cessation of menses for six months, 1 defined it as no menses within 15 months, and 1 defined it as no menses within one year of treatment. Seven earlier trials did not use tamoxifen as adjuvant hormonal therapy, while others did. All of the included patients were stages II-III. The median time of follow-up ranged from 4 to 9 years. HRs for DFS and OS could be extracted from 11 and 5 of the studies, respectively. Most of the survival data were adjusted for a wide range of potential confounders including age, tumor size, HR status, node status and treatment regimen. Detailed information from each trial was listed in Table S1.

\section{Table 1. Main Results of Meta-analysis}

\begin{tabular}{|c|c|c|c|c|}
\hline \multirow{2}{*}{$\frac{S}{\text { Overall Survival (OS) }}$} & \multicolumn{2}{|c|}{$\begin{array}{l}\text { No.of } \\
\text { studies }\end{array}$} & Heterogeneity & $\begin{array}{l}\text { eneity } \\
\text {-value }\end{array}$ \\
\hline & & & & \\
\hline All studies & 5 & $0.69(0.52-0.91)$ & $51.6 \%$ & 0.067 \\
\hline \multicolumn{5}{|l|}{ Definition of CIA } \\
\hline CIA $\geq 6$ months & 3 & $0.72(0.62-0.85)$ & $0.0 \%$ & 0.372 \\
\hline \multicolumn{5}{|l|}{ HR status } \\
\hline HR-negative & 3 & $0.69(0.51-1.00)$ & $0.0 \%$ & 0.902 \\
\hline HR-positive & 3 & $0.54(0.35-0.84)$ & $43.5 \%$ & 0.170 \\
\hline \multicolumn{5}{|l|}{ Disease-Free-Suvival (DFS) } \\
\hline All studies & 11 & $0.74(0.64-0.86)$ & $43.2 \%$ & 0.048 \\
\hline \multicolumn{5}{|l|}{ Definition of CIA } \\
\hline CIA $\geq 3$ months & 5 & $0.74(0.55-0.99)$ & $62.9 \%$ & 0.019 \\
\hline CIA $\geq 6$ months & 4 & $0.70(0.60-0.81)$ & $0.0 \%$ & 0.646 \\
\hline \multicolumn{5}{|c|}{ Hormone receptor (HR) status } \\
\hline HR-negative & 5 & $0.83(0.64-1.07)$ & $7.1 \%$ & 0.366 \\
\hline HR-positive & 6 & $0.65(0.53-0.80)$ & $41.3 \%$ & 0.130 \\
\hline \multicolumn{5}{|l|}{ Premenopausal status } \\
\hline Last menses $<6$ weeks & 2 & $0.57(0.34-0.95)$ & $41.6 \%$ & 0.056 \\
\hline Last menses $<6$ months & 3 & $0.76(0.65-0.89)$ & $0.0 \%$ & 0.539 \\
\hline Last menses $<12$ months & as 3 & $0.81(0.54-1.22)$ & $75.6 \%$ & 0.017 \\
\hline \multicolumn{5}{|l|}{ Follow up } \\
\hline$\geq 5$ years & 9 & $0.71(0.62-0.82)$ & $30.0 \%$ & 0.161 \\
\hline$<5$ years & 2 & $1.01(0.51-2.00)$ & $80.3 \%$ & 0.024 \\
\hline \multicolumn{5}{|l|}{ Tamoxifen use } \\
\hline Yes & 5 & $0.78(0.63-0.97)$ & $32.3 \%$ & 0.206 \\
\hline No & 7 & $0.64(0.48-0.86)$ & $80.1 \%$ & 0.000 \\
\hline \multicolumn{5}{|l|}{ Country } \\
\hline Western & 9 & $0.73(0.62-0.86)$ & $51.1 \%$ & 0.025 \\
\hline Eastern & 2 & $0.82(0.54-1.25)$ & $0.0 \%$ & 0.498 \\
\hline \multicolumn{5}{|l|}{ Treatment Regimen } \\
\hline AT-based regimens & 2 & $0.72(0.60-0.85)$ & $0.0 \%$ & 0.330 \\
\hline CMF-based regimens & 8 & $0.70(0.58-0.84)$ & $43.3 \%$ & 0.070 \\
\hline \multicolumn{5}{|l|}{ Study Design } \\
\hline Retrospective & 4 & $0.61(0.46-0.81)$ & $8.3 \%$ & 0.360 \\
\hline Prospective & 7 & $0.78(0.66-0.92)$ & $50.9 \%$ & 0.047 \\
\hline
\end{tabular}

$\overline{\mathrm{CMF} \text {, cyclophosphamide/methotrexate/fluorouracil; TA, docetaxel/ }}$ doxorubicin 
Meta analysis

Data for DFS were available from all 11 trials (Ludwig Breast Cancer Study Group., 1985; Goldhirsch et al., 1990; Bianco et al., 1991; Budman et al., 1998; Pagani et al., 1998; Parulekar et al., 2005; Colleoni et al., 2006; Jung et al., 2010; Swain et al., 2010; Ganz et al., 2011; Park et al., 2012) with 8333 patients reported. The CIA group was associated with a statistically significant $26 \%$ improvement in DFS when compared with the control group $\left(\mathrm{HR}=0.74,95 \% \mathrm{CI} 0.64-0.86 ; \mathrm{I}^{2}=43.1 \%\right)$ (Figure 1) under a random-effect model. OS was reported in 5 trials (Tormey et al., 1992; Budman et al., 1998; Parulekar et al., 2005; Tierney et al., 2007; Swain et al., 2010) of the 11 trials, including 4193 patients. The efficacy of CIA on the overall survival were presented in these trials $\left(\mathrm{HR}=0.69,95 \% \mathrm{CI} 0.52-0.91 ; \mathrm{I}^{2}=51.6 \%\right.$, random-effects model) (Figure 2). The heterogeneity dropped after removing 1 trials $\left(\mathrm{I}^{2}=0.0 \%\right)$ (Budman et al., 1998), and due to relative small sample size, we should cautiously come to a conclusion.

\section{Subgroup analysis}

According to the definition of CIA, 5 studies defined it as cessation of menses for three months, and the pooled HR of these trials for DFS showed no statistical significance (HR $=0.74,95 \% \mathrm{CI} 0.55-0.99)$. Furthermore, 4 trials (Tormey et al., 1992; Jung et al., 2010; Swain et al., 2010; Park et al., 2012) reported HRs for DFS in the CIA $\geq 6$ months subgroup. The pooled HRs favoured the CIA group (HR=0.70, 95\%CI 0.60-0.81). However, only three in the CIA $\geq 6$ months subgroup reported HR for OS , and the pooled HR was 0.72 (95\%CI 0.62-0.85), which corresponds to a $28 \%$ reduction in the risk of death compared with control group.

When taken hormone receptor (HR) status into consideration, a significant prognostic role of CIA on breast cancer was detected in the patients with hormone receptor-positive (ER+ and/or PR+). The pooled HRs for DFS and OS in hormone receptor-positive subgroup were 0.65 (95\%CI $0.53-0.80)$ and 0.54 (95\%CI $0.35-0.84$ ), respectively (Figure 3$)$. However, no statistical significance reached in the Hormone receptor-negative (ER- and PR-) subgroup for DFS and OS (HR=0.83, 95\%CI 0.64-1.07; $\mathrm{HR}=0.69$, 95\%CI 0.51-1.00, respectively) (Figure 3). In the subgroup analyses on disease-free survival, 7 studies used tamoxifen as adjuvant therapy for breast cancer patients and the remaining 4 studies did not. The summary HR estimates for both subgroups showed significant correlations with DFS (HR $=0.78,95 \% \mathrm{CI}$ $0.63-0.97 ; \mathrm{HR}=0.64,95 \% \mathrm{CI} 0.48-0.86$, respectively). When the definition of premenopausal status was taken into consideration, the pooled survival data showed an favorable survival prognosis in last menses $<6$ weeks and last menses $<6$ months subgroups $(\mathrm{HR}=0.57,95 \% \mathrm{CI}$ 0.34-0.95; HR=0.76, 95\% CI 0.65-0.89, respectively), but not in the last menses $<12$ months subgroup (HR $=0.81$, 95\% CI 0.54-1.22). When stratified by median follow-up, the combined HRs of follow up $\geq 5$ years and follow up 5 years were 0.71 (95\%CI 0.62-0.82) and 1.01 (95\%CI $0.51-2.00)$, respectively. When grouped by the region, the pooled HR of nine studies from western countries showed a statistically significant effects on DFS (HR=0.73,95\% CI 0.62-0.86). However, the combined HR of the other two studies from eastern country showed no statistically

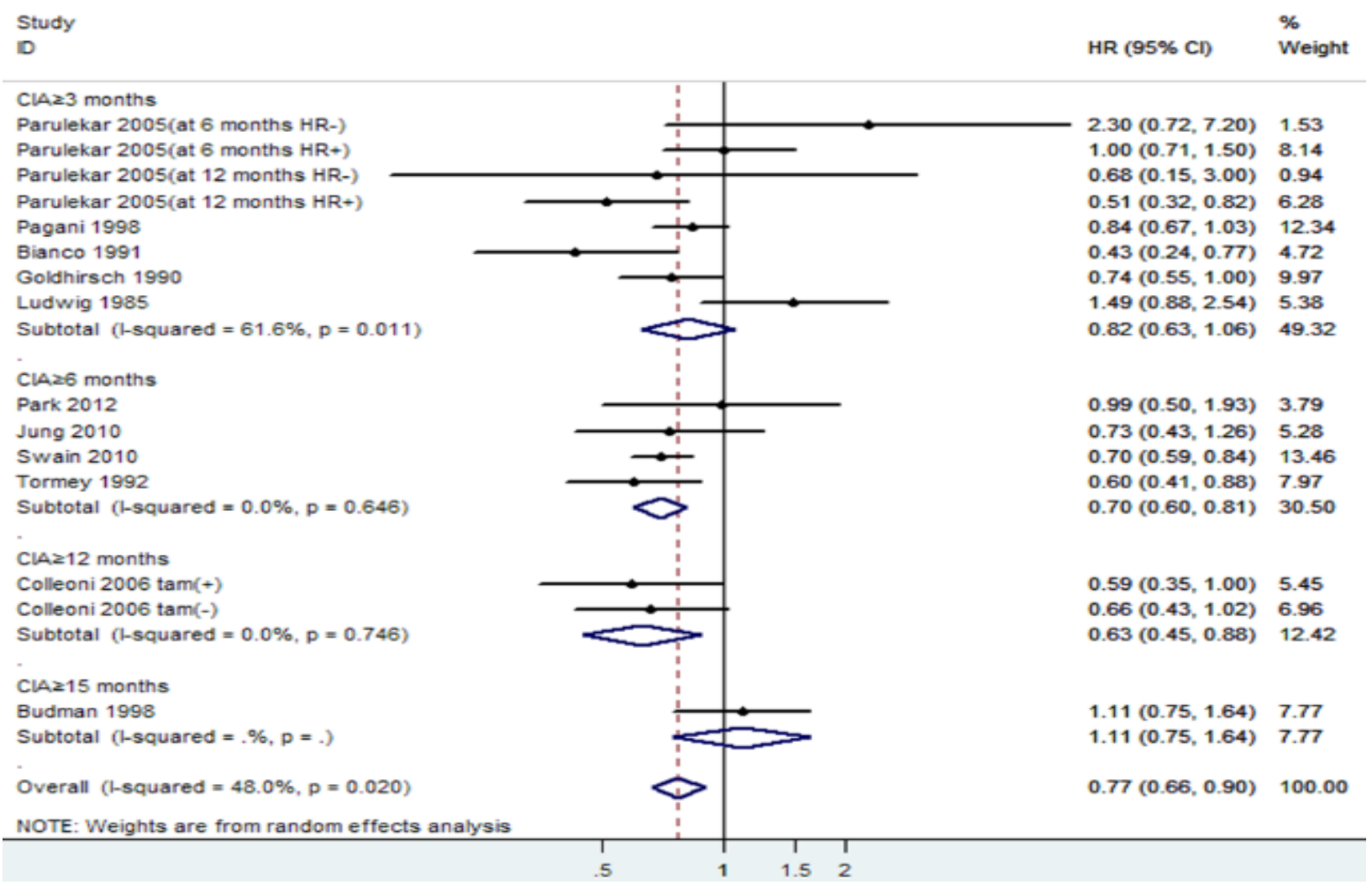

Figure 1. The Association between CIA and Disease-free Survival (DFS) of Breast Cancer Stratified by the

Definition of CIA. The summary HR and 95\% CIs were shown (from the random-effects model) 
Quan Bin Zha et al

significant difference $(\mathrm{HR}=0.82,95 \% \mathrm{CI} 0.54-1.25)$. In the subgroup analysis by treatment regimen, both AT-based regimens and CMF-based regimens showed a statistically significant effects on DFS (HR=0.72, 95\%CI 0.60-0.85; $\mathrm{HR}=0.70,95 \% \mathrm{CI} 0.58-0.84$, respectively). When grouped by study design, the pooled HR of four retrospective studies and seven prospective studies were 0.61 (95\% CI $0.46-0.81)$ and 0.78 (95\%CI 0.66-0.92), respectively.

\section{Publication bias}

Visual assessment of the funnel plot did not provide evidence of publication bias for studies in the DFS outcome. The Begg rank correlation test and Egger linear regression test also indicated no evidence of publication bias (Begg, $p=0.66$; Egger, $p=0.57$ ).

\section{Discussion}

There is still a great deal of controversy with regard to the role of CIA in predicting prognosis and selecting chemotherapy regimen in breast cancer patients. To confirm whether CIA can serve as a useful factor in predicting clinical effects of systemic adjuvant chemotherapy, we undertook a systematic review and meta-analysis of the literature. The results of our meta-analysis showed that CIA was correlated with significant reduction in the risk

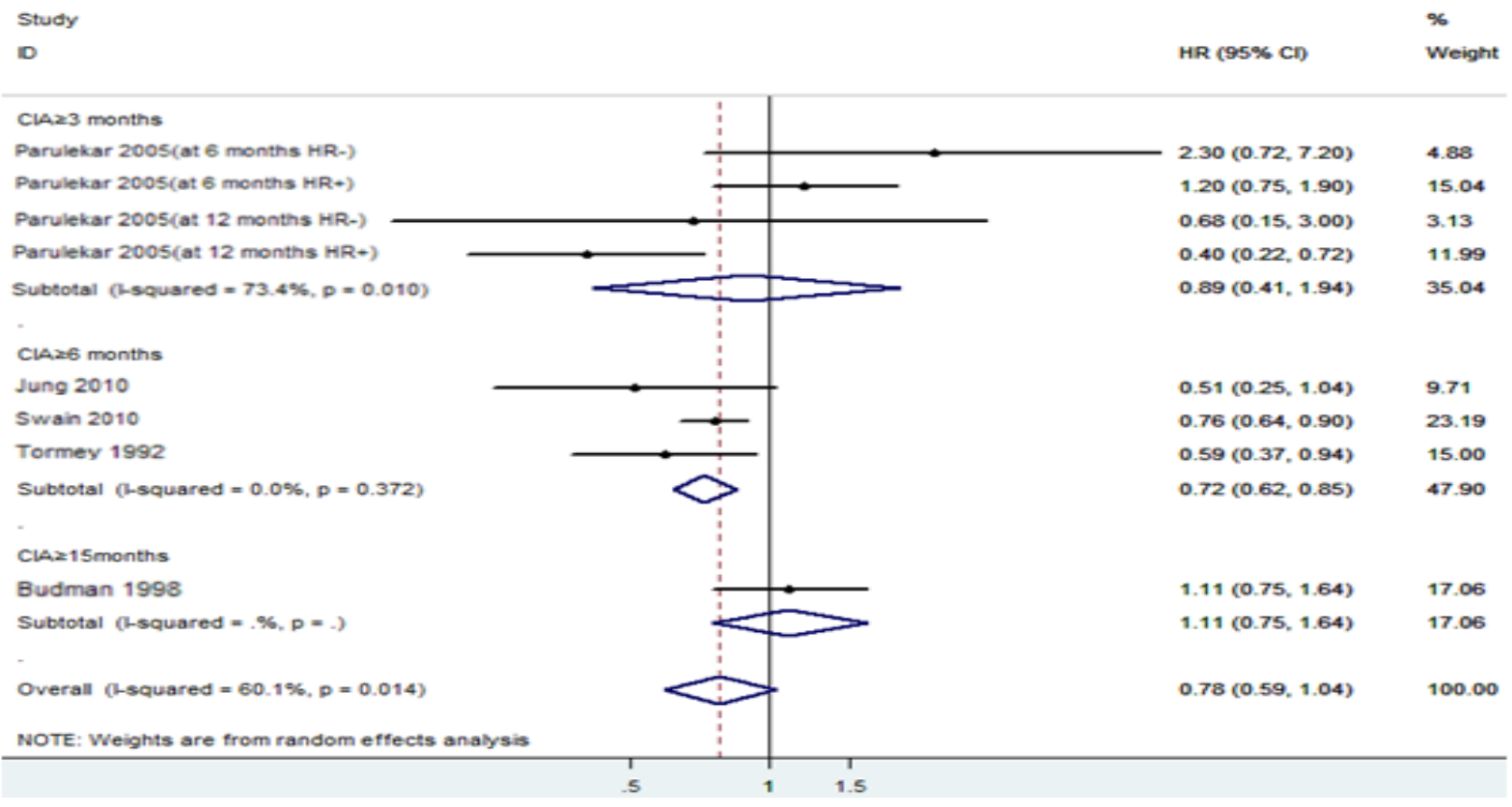

Figure 2. Forest Plot Showing the Combined HR from the Random-effects Model for OS Grouped by the Definition of CIA

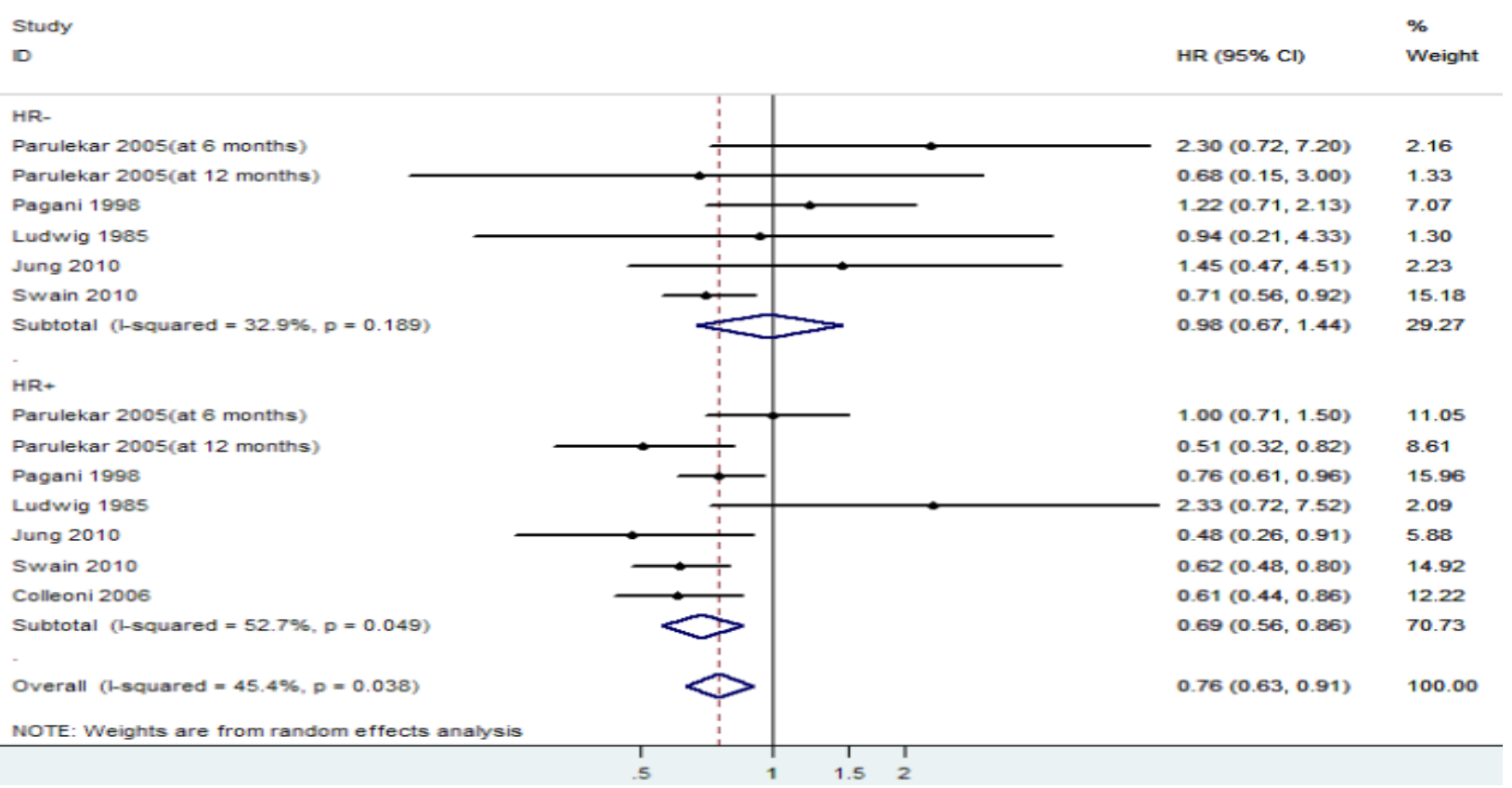

Figure 3. Forest Plot Showing the Combined HR from the Random-effects Model for DFS Grouped by Hormone Receptor (HR) Status 
of recurrence $(\mathrm{HR}=0.74,95 \% \mathrm{CI} 0.64-0.86)$. When the five studies reported the HR of overall survival were pooled, a favorable trend was found $(\mathrm{HR}=0.69,95 \% \mathrm{CI} 0.52-0.91)$. However, a significant heterogeneity was noticed $\left(\mathrm{I}^{2}=\right.$ $51.6 \%)$. When one study by Parulekar et al. was excluded, the heterogeneity dropped. Moreover, the pooled HRs for DFS and OS all favored CIA when it definted as cessation of menses for six months with no heterogeneity $\left(\mathrm{I}^{2}=0.0 \%\right)$. However, the benefit of CIA on OS was uncertain because of less available trials reporting HRs.

The results of subgroup analysis also indicated that there were significantly gains in DFS and OS in the patients with hormone receptor-positive status, but not in the patients with hormone receptor-negative status. These results suggested that benefits of chemotherapy were partially attributed to an indirect endocrine effect in hormone-sensitive tumors.

When we pooled the HR for overall survival of five studies, a significant heterogeneity was found $\left(\mathrm{I}^{2}=\right.$ $51.6 \%$ ). When one study by Parulekar et al was excluded, the heterogeneity declined. The heterogeneity was probably due to the difference in defining amenorrhea and menopause, duration of amenorrhea, the duration of follow-up, chemotherapeutic regimen and characteristics of patients (age, tumor stage, hormone receptor status, race or country). For instance, when we stratified them according to the definition of CIA, Strong heterogeneity existed in the CIA $\geq 3$ months subgroup analysis for DFS. Lack of standardized data collection including bleeding history and long menstrual cycle may partially explain this heterogeneity. In addition, different detecting methods for hormone receptor may also contribute to the heterogeneity in hormone receptor-positive subgroup $\left(\mathrm{I}^{2}=\right.$ $41.3 \%$ for DFS; $\mathrm{I}^{2}=43.5 \%$ for OS). Taking heterogeneity into consideration, we used a random-effects model for more conservative estimates. Nevertheless, there is no conclusive interpretation for the heterogeneity.

Potential limitations of this study should be considered. First, the characteristics of these trials including the defining amenorrhea and menopause, duration of amenorrhea, the duration of follow-up, and characteristics of patients were varied. Second, our study was based on abstracted data and not on individual patient data, which may not provide robust estimation for the HRs and 95\%CIs. Third, the quality of eligible studies influences the reliability of our meta-analysis remarkably. Published articles often provide insufficient information to assess the quality of the study. Fourth, publication bias may be unavoidable in this article. However, little evidence of publication bias was observed. Five, although most of studies adjusted for a series of key covariates, we still cannot rule out the possible residual confounding variables. Six, the total number of included studies and the total sample size were relatively small, which may influence the accuracy of meta-analysis to some extent. Seven, most chemotherapy regimens were CMF-based regimens, although, the incidence of amenorrhea with anthracycline-taxane-containing chemotherapy regimens and its impact on survival were similar. Finally, the majority of studies were carried out in western countries, and additional research in other populations is warranted to generalize the findings.

Our study still has important clinical implications. It showed that premenopausal patients developing amenorrhea during chemotherapy had a significantly better DFS and OS compared to those who did not. Moreover, the subgroup analysis demonstrated that CIA was correlated with longer DFS and OS in hormone receptor-positive subgroup. However, the correlation between CIA and OS need to be investigated, because of limited data. These results strongly suggest that endocrine manipulation through ovarian suppression is another mechanism of chemotherapy in addition to direct cytotoxicity in women with hormone-sensitive disease. Ovarian suppression, induced either by surgery, or LH-RH superanalogues therapy, has been shown to be an effective treatment in breast cancer patients (Early Breast Cancer Trialists' Collaborative Group., 1992). On this basis, we can suppose that the better outcome of patients with CIA is related to the combined action of two effective treatments: chemotherapy and ovarian ablation. The hypothesis of a double effect, cytotoxic and endocrine, could explain the greater impact of chemotherapy generally observed in premenopausal women with hormone-sensitive breast cancer (Li Xiu-Juan et al., 2014). This inclusion was indirectly supported by two large randomized prospective trials (Jakesz et al., 2002; Jonat et al., 2002). Because of serious climacteric disturbances such as vasomotor symptoms and urogenital atrophy in young women, physicians should avoid artificially inducing amenorrhea in premenopausal patients without selection (Yu Ke-Da et al., 2014). In conclusion, a comprehensive understanding of the impact of CIA on survival in premenopausal breast cancer patients is critical (Zhao Jianli et al., 2014). In order to draw definite conclusions, multicenter randomized prospective trial of adjuvant chemotherapy should evaluate CIA using exact definitions of CIA and premenopausal status.

\section{References}

Beex LV, Mackenzie MA, Raemaekers JM, et al (1988). Adjuvant chemotherapy in premenopausal patients with primary breast cancer; relation to drug-induced amenorrhoea, age and the progesterone receptor status of the tumour. Eur J Cancer Clin Oncol, 24, 719-21.

Begg CB, Mazumdar M (1994). Operating characteristics of a rank correlation test for publication bias. Biometrics, $\mathbf{5 0}$, 1088-101.

Bianco AR, Del Mastro L, Gallo C, et al (1991). Prognostic role of amenorrhea induced by adjuvant chemotherapy in premenopausal patients with early breast cancer. $\mathrm{Br} J$ Cancer, 63, 799-803.

Borde F, CMI, Fumoleau P, et al (2003). Role of chemo-induced amenorrhea in premenopausal, node-positive, operable breast cancer patients: 9-year follow-up results of French Adjuvant Study Group data base. Breast Cancer Res Treat, 82, 30.

Brincker H, Rose C, Rank F, et al (1987). Evidence of a castrationmediated effect of adjuvant cytotoxic chemotherapy in premenopausal breast cancer. J Clin Oncol, 5, 1771-8.

Budman DR, Berry DA, Cirrincione CT, et al (1998). Dose and dose intensity as determinants of outcome in the adjuvant treatment of breast cancer: The Cancer and Leukemia Group 


\section{B. J Natl Cancer Inst, 90, 1205-11.}

Campora E, Pronzato P, Amoroso D, et al (1992). Prognostic factors in node positive primary breast cancer patients treated with adjuvant CMF. Anticancer Res, 12, 1555-8.

Colleoni M, Gelber S, Goldhirsch A, et al (2006). Tamoxifen after adjuvant chemotherapy for premenopausal women with lymph node-positive breast cancer: international breast cancer study group trial 13-93. J Clin Oncol, 24, 1332-41.

Early Breast Cancer Trialists' Collaborative Group (1992). Systemic treatment of early breast cancer by hormonal, cytotoxic or immune therapy. Lancet, 339, 71-85

Early Breast Cancer Trialists' Collaborative Group (EBCTCG) (2005). Effects of chemotherapy and hormonal therapy for early breast cancer on recurrence and 15-year survival: an overview of the randomised trials. Lancet, 365, 1687-717.

Fisher B (1979). 1-phenylalanine mustard (L-Pam) in the management of premenopausal patients with primary breastcancer-Lack of association of disease-free survival with depression of ovarian function. Cancer, 44, 847-57.

Ganz PA, Land SR, Geyer CE Jr, et al (2011). Menstrual history and quality-of-life outcomes in women with node-positive breast cancer treated with adjuvant therapy on the NSABP B-30 trial. J Clin Oncol, 29, 1110-6.

Goldhirsch A, Gelber RD, Castiglione M (1990). The magnitude of endocrine effects of adjuvant chemo-therapy for premenopausal breast cancer patients. Ann Oncol, 1, 183-8.

Higgins JP, Thompson SG, Deeks JJ, et al (2003). Measuring inconsistency in meta-analyses. BMJ, 327, 557-60.

Howell A, Rubens RD, Bush H, et al (1984). A controlled trial of adjuvant chemotherapy with melphalan versus cyclophosphamide, methotrexate, and fluorouracil for breast cancer. Recent Results Cancer Res, 96, 74-89.

Jakesz R, Hausmaninger H, Kubista E, et al (2002). Randomized adjuvant trial of tamoxifen and goserelin versus cyclophosphamide, methotrexate, and fluorouracil: Evidence for the superiority of treatment with endocrine blockade in premenopausal patients with hormone-responsive breast cancer Austrian Breast and Colorectal Cancer Study Group trial 5. J Clin Oncol, 20, 4621-7.

Jonat W, Kaufmann M, Sauerbrei W, et al (2002). Goserelin versus cyclophosphamide, methotrexate, and fluorouracil as adjuvant therapy in premenopausal patients with nodepositive breast cancer:The Zoladex Early Breast Cancer Res Association Study. J Clin Oncol, 20, 4628-35.

Jung M , Shin HJ, Rha SY, et al. (2010). The Clinical Outcome of Chemotherapy-Induced Amenorrhea in Premenopausal Young Patients with Breast Cancer with Long-Term Followup. Ann Surg Oncol, 17, 3259-68.

Leining MG, Gelber S, Rosenberg R, et al (2006). Menopausaltype symptoms in young breast cancer survivors. Ann Oncol, 17, 1777-82.

Li XJ, Ren ZJ, Tang JH (2014). Lack of prognostic value of human epidermal growth factor- like receptor 2 status in inflammatory breast cancer (IBC): a meta-analysis. Asian Pac J Cancer Prev, 15, 9615-9

Ludwig Breast Cancer Study Group (1985). A randomized trial of adjuvant combination chemotherapy with or without prednisone in premenopausal breast cancer patients with metastases in one to three axillary lymph nodes. Cancer Res, 45, 4454-9.

Mastro LD, Venturini M, Sertoli MR, et al (1997). Amenorrhea induced by adjuvant chemotherapy in early breast cancer patients: prognostic role and clinical implications. Breast Cancer Research and Treatment, 43, 183-90.

Padmanabhan N, Rubens RD, Howell A (1986). Adjuvant chemotherapy in early breast cancer. Lancet, 2, 1333-4.

Pagani O, O’Neill A, Castiglione M, et al (1998). Prognostic impact of amenorrhoea after adjuvant chemotherapy in premenopausal breast cancer patients with axillary node involvement: Results of the International Breast Cancer Study Group (IBCSG) Trial VI. Eur J Cancer, 34, 632-40.

Park H, Han H.S, Lee H, et al (2012). Resumption or persistence of menstruation after cytotoxic chemotherapy is a prognostic factor for poor disease-free survival in premenopausal patients with early breast cancer. Ann Oncol, 23, 2283-9.

Parulekar WR, Day AG, Ottaway JA, et al (2005). Incidence and prognostic impact of amenorrhea during adjuvant therapy in high-risk premenopausal breast cancer: Analysis of a National Cancer Institute of Canada Clinical Trials Group Study-NCIC CTG MA. 5. J Clin Oncol, 23, 6002-8.

Richards MA, O'Reilly SM, Howell A, et al (1990). Adjuvant cyclophosphamide, methotrexate, and fluorouracil in patients with axillary node-positive breast cancer: An update of the Guy's/Manchester trial. J Clin Oncol, 8, 2032-9.

Siegel R, Naishadham D, Jemal A (2015). Cancer statistics, 2015. CA Cancer J Clin, 65, 5-29.

Swain SM, Jeong JH, Geyer Jr.CE, et al (2010). Longer therapy, iatrogenic amenorrhea, and survival in early breast cancer. $N$ Engl J Med, 22, 2053-65.

Swain SM, Land SR, Ritter MW, et al (2009). Amenorrhea in premenopausal women on the doxorubicin-andcyclophosphamide-followed-by-docetaxel arm of NSABP B-30 trial. Breast Cancer Res Treat, 113, 315-20.

Tierney JF, Stewart LA, Ghersi D, Burdett S, et al (2007). Practical methods for incorporating summary time-to-event data into meta-analysis. Trials, $\mathbf{8}, 16$.

Tobias A (1999). Assessing the influence of a single study in the meta-anyalysis estimate. Stata Technical Bulletin, 8, 15-27.

Tormey DC, Gray R, Abeloff MD, et al (1992). Adjuvant therapy with a doxorubicin regimen and long-term tamoxifen in premenopausal breast cancer patients: an eastern cooperative oncology group trial. J Clin Oncol, 10, 1848-56.

Walshe JM, Denduluri N, Swain SM (2006). Amenorrhea in premenopausal women after adjuvant chemotherapy for breast cancer. J Clin Oncol, 24, 5769-79.

Yazilitas D, Sendur MAN, Karaca H (2015). Efficacy of dose dense doxorubicin and cyclophosphamide followed by paclitaxel versus conventional dose doxorubicin, cyclophosphamide followed by paclitaxel or docetaxel in patients with node-positive breast cancer. Asian Pac J Cancer Prev, 16, 1471-7.

Yu KD, Shao ZM (2014). Survival benefit from response-guided approach: a direct effect of more effective cytotoxic regimens or an indirect effect of chemotherapy-induced amenorrhea? J Clin Oncol, 32, 1282-3.

Zhao JL, Liu JQ, Chen K, et al (2014). What lies behind chemotherapy-induced amenorrhea for breast cancer patients: a meta-analysis. Breast Cancer Res Treat, 145, 113-28. 